\title{
Structural Plasticity in the Drosophila Brain
}

\author{
Martin Heisenberg, Monika Heusipp, and Christiane Wanke \\ Theodor-Boveri-Institut für Biowissenschaften (Biozentrum), Lehrstuhl für Genetik, Am Hubland, D-97074 Würzburg, \\ Germany
}

\begin{abstract}
The Drosophila brain is highly variable in size. Female flies grown in densely populated larval cultures have up to $20 \%$ more Kenyon cell fibers in their mushroom bodies than flies from low-density cultures. These differences in the number of Kenyon cell fibers are accompanied by differences in the volume of the calyx. During imaginal life, volume changes are observed in the calyces, all parts of the optic lobes, the central brain, and central complex. They occur not only in the first week of adulthood but also between days 8 and 16. Factors causing these changes are little understood. In flies kept in pairs for 1 week, the size of the calyx but not of the lobula is influenced by the sex of the partner. Females have larger calyces if the partner is female than if it is male. Males seem to be affected in the same way. Females living solitarily in little food vials for the first week of their imaginal life have smaller lobulae than females having spent the week in a populated flight cage. Males and females of heterosexual groups of $\mathbf{4 0}$ animals have still smaller lobulae, medullae, and calyces than their siblings kept in isolation. In addition, brain size is influenced by yet unidentified factors causing long and short trends. The data suggest that in Drosophila, most neuropil regions of the brain are continuously reorganized throughout life in response to specific living conditions.
\end{abstract}

[Key words: brain volume, experience dependence, mushroom body, optic lobe, influence of space, influence of social contact]

The number of Kenyon cell fibers in the mushroom bodies of adult Drosophila melanogaster depends upon their living conditions. After 3 weeks in groups of 200 , in large $(50 \mathrm{~cm})^{3} \mathrm{flight}$ cages in a circadian light-dark cycle with various odor sources, colored patterns on the walls, plants and daily handling, flies have about $15 \%$ morc Kenyon cell fibers than their "deprived" siblings that have been maintained singly in small plastic vials with standard food in the dark (Technau, 1984). This finding raises three immediate questions. (1) What happens at the cellular level? (2) What are the effective parameters in the environment during "deprivation" and "enrichment." (3) What is

\footnotetext{
Received June 8, 1994; revised Aug. 15, 1994; accepted Sept. 1, 1994.

We are indebted to $\mathrm{R}$. Wolf who provided the computer program for planimetric evaluation of brain sections, to R. Flagner, J. Roth, and C. Wolf for their help collecting data, to R. Wolf and $\mathrm{C}$. Wolf for preparing the figures, to M. Dill for help with the statistics, to S. Schneuwly, G. O. Pflugfelder, and one of the reviewers for critical comments on the manuscript, to I. C. Hall for encouragement, to Deutsche Forschungsgemeinschaft (Grant He 986), and the Human Frontiers Science Program for support.

Correspondence should be addressed to Martin Heisenberg at the above address.

Copyright (C) 1995 Society for Neuroscience 0270-6474/95/151951-10\$05.00/0
}

the behavioral correlate of this structural plasticity (if there is any)?

Technau (1984) observed a pronounced increase in fiber number during the first week of adulthood, no change in the second week, and a slow decline in the third. The increase was accompanied by low-level mitotic activity in the cellular cortex of the calyx which, however, appeared to be too small to account for the increase in fiber number during that period (see also Ito and Hotta, 1992). In a follow-up study, Balling et al. (1987) observed, that in the same wild-type stock the fiber number in newly eclosed flies was markedly higher than recorded earlier and that it declined during the first week. Obviously, increase and decline both can happen during early adulthood, depending upon the size of the mushroom bodies at eclosion. This situation is reminiscent of the degeneration and regrowth of Kenyon cell fibers during early metamorphosis (Technau and Heisenberg, 1982). Cell bodies are not affected during this process of reorganization. Probably also during early adulthood fiber outgrowth and regression occur independently of cell proliferation and cell death.

Little is known about the effective variables of "deprivation" and "enrichment." Technau (1984) dismissed visual deprivation as a major cause. Confinement in small food vials is sufficient to render mushroom bodies small even under a circadian lightdark regime. These results are in line with known anatomical and behavioral data: the mushroom bodies are part of the olfactory pathway and figure importantly in olfactory conditioning (e.g., Heisenberg et al., 1985, deBelle and Heisenberg, 1994; for the honey bee: Erber et al., 1980; Menzel et al., 1991). They do not appear to receive major direct input from the optic lobes and genetic or pharmacological ablation of the mushroom bodies (Prokop and Technau, 1994) has no obvious effect on visual behavior (Heisenberg et al., 1985, unpublished observations).

Since mushroom bodies are involved in olfactory learning and memory (reviews: Heisenberg, 1989, 1994) the turnover of Kenyon cell fibers might be related to this process. Indeed, olfactory learning mutants (dunce, rutabaga) fail to show this experiencedependent structural plasticity (Balling et al., 1987). On the other hand, as regulators of cAMP concentration these two genes may be involved in various aspects of neuronal and behavioral plasticity. For instance, changes in the basic branching patterns of terminal arborizations of sensory and motor neurons are found in mutants with altered excitability (Budnik et al., 1989, 1990; Corfas and Dudai, 1991). Again, this plasticity is affected by mutations in the dunce and rutabaga genes (Zhong et al., 1992). The behavioral correlate of the fiber turnover in Kenyon cells has yet to be discovered. In the honeybee, experience-dependent structural plasticity in the brain has been reported, too. Morphological differences in the calyx are shown to correlate with the 
division of labor (Coss and Brandon, 1982; Durst et al., 1993; Withers et al., 1993).

For experimental reasons, the three questions posed in the first paragraph have remained largely unanswered. We have now overcome some of the difficulties. The present article describes (1) an improved method of data acquisition; (2) a major source of variability affecting mushroom body size at eclosion from the pupa; (3) altered rearing conditions during the adult phase that improve the long-term reproducibility of results. We further report (4) structural plasticity in most parts of the fly brain; (5) structural plasticity in brains not only of young flies but also of flies between 8 and $16 \mathrm{~d}$ of age; (6) the fly's living space and social environment to be important parameters influcncing brain structure.

Activity-dependent structural plasticity of the brain is well documented for vertebrates (e.g., Cotman et al., 1987; Thompson, 1987) and some invertebrates (review: Bailey and Kandel, 1993). Nevertheless, the arthropod brain is still regarded as largely "hard-wired," due to the highly and precisely repetitive, neurocrystalline optic lobes and the abundance of interindividually identifiable neurons in the CNS (for the fly: e.g., Strausfeld, 1976). The present study is concerned exclusively with the Drosophila brain. However, our findings with this species may well be representative of all arthropods. Structural plasticity may turn out to be a property of all brains, enabling ambulatory organisms to adjust to slow and steady changes of their living conditions throughout life.

\section{Materials and Methods}

Wild-type Berlin was used throughout. If not stated otherwise, flies were allowed to deposit eggs for up to $9 \mathrm{hr}$ on petri dishes containing 5\% sucrose in $3 \%$ agar, supplemented with one drop of a suspension of fresh brewer's yeast per plate. In the experiments of part I, the indicated numbers of eggs or embryos were transferred to $70 \mathrm{ml}$ bottles with 10 $\mathrm{ml}$ standard food (corn meal, molasses, no fresh yeast). In part II, 250 animals were transferred to $50 \mathrm{ml}$ of food in $180 \mathrm{ml}$ bottles. Pupae were transferred to their respective containers 7-10 d after egg laying with a wet brush, as indicated in the Results section. Before March 1993, the imagines in food vials and in the flight cage were all kept in the same climatized fly room. Cultures were kept at $23.5^{\circ} \mathrm{C}, 60 \%$ relative humidity, in a 12L:12D cycle. From March 1993 on (Figs. 6-10), all animals, whether in food vials or not, were kept in an acrylic glass incubator $\left[(50 \mathrm{~cm})^{3}\right]$ in a partially controlled atmosphere. The incubator was supplied with air from a central compressor, filtered through $200 \mathrm{ml}$ of charcoal and two wash bottles with deionized water. Temperature in the incubator was $24^{\circ} \mathrm{C}$ at $90 \%$ relative humidity. The daily light cycle was $16 \mathrm{~L}: 8 \mathrm{D}$. After the indicated period, the incubator and food vials were transferred to $4^{\circ} \mathrm{C}$ for $20 \mathrm{~min}$. This was enough to immobilize all animals allowing collection for histology. Hlies were always collected between 0930 and $1030 \mathrm{hr}$.

Standard techniques for electron microscopy and mass histology (Ashburner, 1989b; Technau, 1984) were used. Evaluation of fiber number in the dorsal peduncle has been described in Technau and Heisenberg (1982). Paraffin sections were inspected by fluorescence microscopy. Volumes of neuropil regions were evaluated by planimetry aided by computer video and a special program developed and kindly provided by $R$. Wolf. Measuring errors were small compared with the interindividual differences among flies of the same group. Flies of both sexes and living conditions were arranged in random order on the microscope slides. Brain size and fiber number were evaluated without knowledge of the sex or living conditions of the respective flies.

\section{Results}

Size of mushroom bodies at pupal eclosion

Mushroom bodies of newly hatched adult female flies (wild-type Berlin, WTB) have between 2400 and 3000 Kenyon cell fibers per peduncle. Previous studies (Balling et al., 1987) had shown that this large variability depends in part upon factors that affect

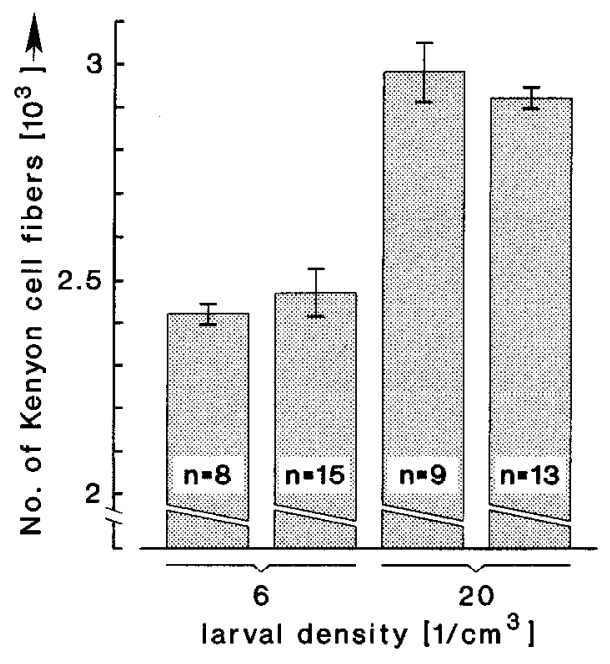

Figure 1. Number of Kenyon cell fibers in the mushroom bodies of females at eclosion depends upon density of larval cultures. Fibers were counted in electron micrographs of peduncle cross sections about 30 $\mu \mathrm{m}$ anterior to the calyx. Larval densities are given for the total volume of food $(10 \mathrm{ml})$, although the animals stayed at the upper surface. The experiment was replicated after 5 months to give the second set of counts in each group. Differences between high- and low-density cultures in both experiments are highly significant $(p<0.001) . n$ indicates the number of peduncles and animals. Error bars in this and all following figures are SEMs.

all the flies of a given population in common. In other words, the standard deviation of a given group average is much smaller than would be expected from the overall variation. We considered three possible explanations: (1) a circannual trend, (2) extrinsic influences on the larval culture (failure of temperature or humidity control), (3) intrinsic factors of the larval culture (quality of food, diseases, microfauna, population density). Explanations (1) and (2) at that time were not supported by a careful evaluation of the protocols. Since little could be done about diseases and variations in the microfauna, we measured the influence of the density of the larval culture on mushroom body size.

Embryos from a common population were transferred to small vials containing $10 \mathrm{ml}$ of standard food. One vial received 60 animals, the other 200 . Within $1 \mathrm{hr}$ after hatching from the pupal case female flies were sacrificed for electron microscopy. Figure 1 shows the result of two such experiments conducted 5 months apart. Obviously, the density of the larval culture has a profound effect on the number of Kenyon cell fibers in the peduncle. Flies that had lived as larvae under crowded conditions have larger mushroom bodies than their siblings grown with ample space and food supply.

In order to decide whether this effect was due to direct contact between the animals or was mediated by diffusible substances produced or consumed by them, we constructed two double chambers. In one of these the two compartments were separated by filter paper, in the other one by a glass plate that prevented diffusion. To each compartment 5 or 25 embryos $/ \mathrm{ml}$ of food were added. The densely populated food, in addition, contained a food dye to monitor the diffusion between the two chambers separated by the filter paper. The result of this experiment is shown in Figure 2. Diffusion, indeed, abolishes the difference in fiber number between the two groups of flies. A diffusible factor, therefore, must be responsible for the effect. The exper- 


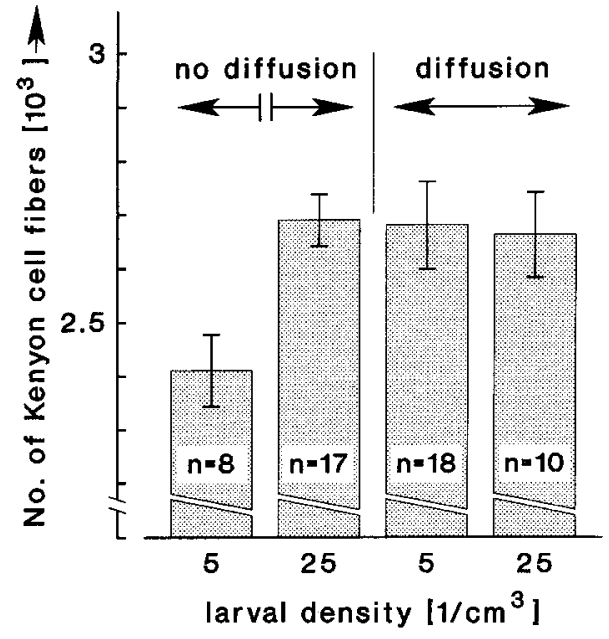

Figure 2. A diffusible factor is responsible for the effect of larval crowding on the number of Kenyon cell fibers in the peduncle at eclosion. To demonstrate this, two identical double chambers were constructed; each consisted of two acrylic glass tubes (diameter, $50 \mathrm{~mm}$; height, $50 \mathrm{~mm}$ ), in one case separated by a Nylon membrane (Pale, pore size $0.45 \mu \mathrm{m}$ ), in the other by a $1 \mathrm{~mm}$ acrylic glass plate. The far ends of the tubes were closed by regular foam rubber stoppers. Standard fly food was poured into each tube to form a $10 \mathrm{~mm}$ layer on either side of the membrane or plate. After cooling to room temperature, the indicated numbers of embryos were added to the compartments. Both double chambers were rolated with three revolutions per hour around a transverse axis to prevent the food from flowing off the membrane or plate. $n=$ number of peduncles (and animals) sampled. Female flies were sacrificed for histology within $\mathbf{l ~ h r}$ of emergence. Without diffusion between the high- and low-density cultures, fiber number was significantly different $(p<0.01)$. With diffusion, no significant difference was observed.

iment cannot distinguish whether this factor acts to enhance or suppress fiber number, for example, between the effect of a pheromone secreted by the animals (which might stimulate Kenyon cell proliferation or fiber outgrowth) and the depletion of inhibitory substances in the food.

Larval crowding is known to have many effects (reviewed in Ashburner, 1989a). It leads to reduced body weight in the adult phase, slows down development, and increases the variability of developmental time, the smallest animals being the latest to eclose. To investigate whether rate of development and mushroom body size are (negatively) correlated, well synchronized embryos ( $0-2 \mathrm{hr}$ of age) were transferred to fresh food vials. In the low-density culture, all flies eclosed from the pupal case on day 13 (at $23.5^{\circ} \mathrm{C}$ ). In the high-density vials, only about $20 \%$ of the flies hatched on this day, about $50 \%$ on day $14,25 \%$ on day 15 , and the few remaining flies on day 16 . This distribution was about the same for males and females. In order to save time and labor, for the evaluation of mushroom body size a different procedure was introduced. The volume of the calyx was measured planimetrically in serial paraffin sections of mass histological preparations (Heisenberg and Böhl, 1979). Considering that the dendritic arborizations of the Kenyon cells contribute prominently to the volume of the calyx, we assumed that changes in the number of Kenyon cell fibers should show in the calyx as well. Indeed, a large volume difference in the female calyx was observed between the high- and low-density larval cultures (Fig. 3 ). In this experiment, the calyces of male and female flies as well as the animals eclosed on different days were evaluated separately. (Note that in the experiments of Figs. 1 and 2 only

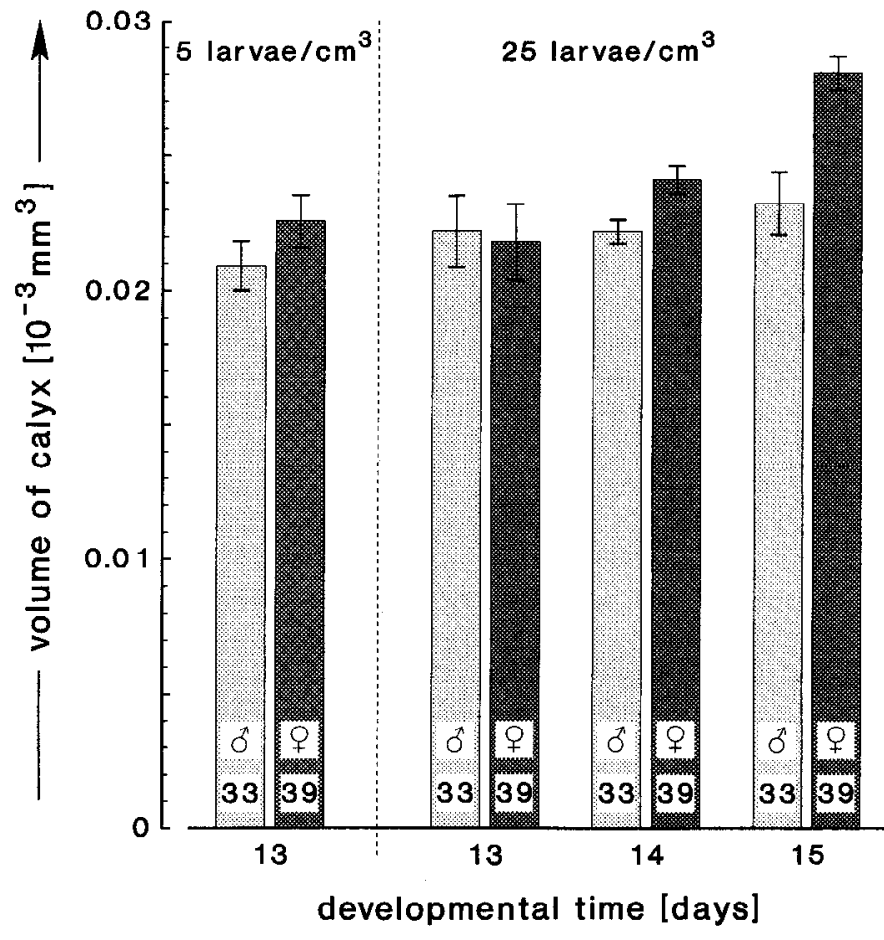

Figure 3. In females, but not in males, calyx size is positively correlated with developmental time. Synchronized cultures of 5 and 25 larvae per $\mathrm{ml}$ of food were raised under otherwise identical conditions. (Difference between 15-d-old females and each of the other female groups is highly significant with $p<0.001$.) The number of calyces evaluated is indicated as a number at the bottom of each bar $(n)$. The number of flies is slightly larger than $n / 2$. No significant correlation is observed between the size of the right and the left calyx of the same brain.

females had been measured.) Interestingly, larval crowding seems to affect the size of the calyx only in females but not (or only slightly) in males. Furthermore, size increases with increasing developmental time.

Shortage of food increases the duration of the larval period (feeding stage) and has comparatively little effect on the rate of development in the pupa (e.g., Sang, 1949). To explain the effect on mushroom body size, one might assume that the mushroom body neuroblasts maintain their high mitotic rate in spite of short supplies. This simplistic view, however, does not account for the sexual specificity of the effect. Shortage of food slows down development of males and females alike. Moreover, considering that in contrast to most other neurons in the brain Kenyon cells have to regenerate their entire fibers during metamorphosis, food shortage might be expected to affect the development of the mushroom bodies more severely than other tissues.

A tentative ecological explanation can be offered for this phenomenon: as long as plenty of food is available, sufficient for the next generation, it must be advantageous for a larva to reach maturity as fast as possible, in order to have its own offspring consume as much of the food as will be left. If, in contrast, the larvae already experience a food shortage, there is no need for them to emerge first, since for the offspring, new food sources will have to be found anyway. For finding suitable egg deposition sites, female flies will have to explore new food sources. Large mushroom bodies may be beneficial for this task. Therefore, we would like to assume that the food situation during the larval period serves as a developmental switch, shifting the de- 
velopmental program of the mushroom body into a direction that prepares the animal for its forthcoming behavior.

Whatever the right interpretation of this phenomenon may be, in females fiber number in the mushroom bodies is positively correlated with developmental time and negatively with body weight. Knowing this relation, one can now manipulate the size of the mushroom bodies at eclosion by natural means.

\section{Structural plasticity of the adult brain}

Structural plasticity occurs in many if not all parts of the brain. With the new planimetric method for assessing the volume of well-discernible regions of neuropil, one now can extend the investigation to other parts of the brain. We considered this as a control and were prepared to find no or very little plasticity in most other regions since the ability of the Kenyon cell axons to regress and then regrow from the old cell bodies, as observed during metamorphosis, was supposed to be a specialty of this particular brain region.

First, the original experiment of Technau (1984) was essentially repeated. Embryos were transferred to fresh food vials at a density of five animals $/ \mathrm{ml}$. Shortly before eclosion, pupae were placed either singly into small isolation vials or as a group of 200 into a flight cage. Single-fly vials were kept under a moist towel in the regular fly room, and flies were transferred to fresh vials twice a week. In the flight cage, food vials and odor sources were also exchanged twice a week, and flies were chased around twice a day for several minutes except on weekends.

After $19 \mathrm{~d}$, female flies of both groups were sacrificed for mass histology. The following regions of neuropil were measured: calyx, optic lobe without lamina, central brain without calyces and optic lobes, the central complex without protocerebral bridge. In addition, in a subset of brains the four neuropil regions of the optic lobes, lamina, medulla, lobula, and lobula plate were evaluated separately. The results are shown in Figure 4. Of all the brain regions studied, the calyx showed the largest difference. Calyces of the flies from the flight cage were $21 \%$ larger than the calyces of flies kept in isolation. Ilowever, lamina and medulla with about $18 \%$ difference followed closely behind. The two groups had a volume difference of $9.5 \%$ in the lobula and about $7.5 \%$ in the whole central brain. The smallest effect was observed in the central complex $(6.5 \%)$ (although the central brain without the peduncles and lobes of the mushroom bodies may have been just as low). For comparison, in a different set of flies kept under the same two conditions, the transverse diameter of the head capsule and body weight were measured. The difference in head diameter was less than $0.5 \%$ and not significant. In each group, 105 females together had the same weight of $140 \mathrm{mg}$ (data not shown in Fig. 4).

Since special care was taken to distribute the pupae of the culture bottles evenly into the two groups, it is assumed that average brain size in the two groups was the same at the onset of the imaginal period. Moreover, in both groups mortality during the $19 \mathrm{~d}$ was extremely low. We, therefore, conclude that the flies' brains had undergone structural changes during that period. Life in the flight cage had led to more neuropil in most parts of the brain, than life in isolation in small tubes. Different parts of the brain were affected to different degrees. What we did not know at this stage of the investigation was that we had only partial control of the parameters causing these differences (see below).

Is the plasticity confined to a critical period? In the earlier
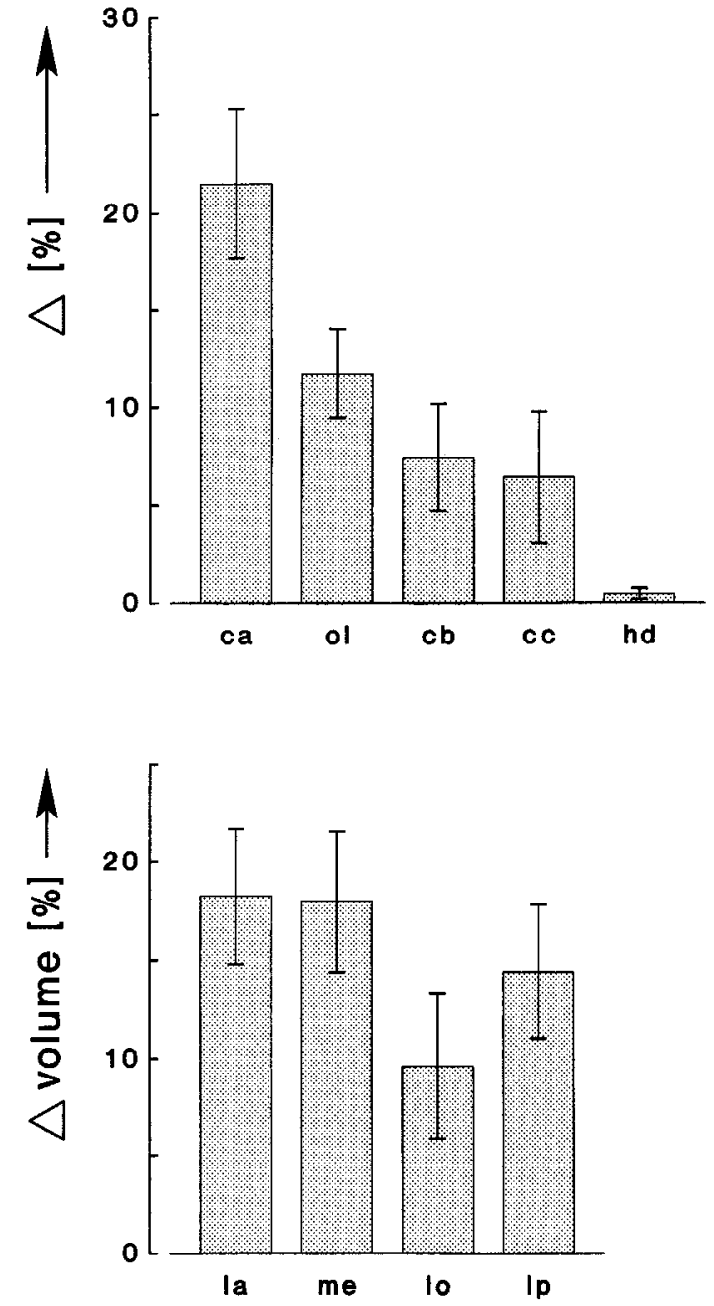

Figure 4. Differences in volume of brain ncuropil regions and head diameter, of female flies after $19 \mathrm{~d}$ of "deprived" and "enriched" living conditions. For details of rearing conditions, see Results. Positive differences indicate that the values of the flies from the "enriched" environment are larger than those of the "deprived" flies. Error bars are SEMs of the differences, calculated from the SEMs of the two respective groups by error propagation. $c a$, calyx; ol, optic lobe; $c b$, central brain; $c c$, central complex; $h d$, head diameter; la, lamina; me, medulla; $l o$, lobula; $l p$, lobula plate. Sample sizes and significance levels for calyx, $n=67 / 82(p<0.001)$; optic lobe, $n=24 / 26(p<0.001)$; central brain, $n=18 / 17(p<0.01)$; central complex; $n=20 / 20(p<$ 0.05 ); head diameter, $n=51 / 57$ (NS); lamina, medulla, lobula and lobula plate, $n=20 / 20(p<0.001)$ each.

work (Technau, 1984; Balling et al. 1987), structural changes had been observed already in the first week of imaginal life. During that period, fiber number in the peduncle rose or declined monotonically from the eclosion level to the value ultimately supported by the respective living conditions. The data did not give any indication whether reorganization was still possible after the first week. If plasticity were restricted to the onset of imaginal life, it could be viewed as a postponement of the final stages of development into adulthood beyond which reorganization is no longer possible.

In the next experiment (Fig. 5), therefore, flies were treated for $8 \mathrm{~d}$ in the manner described above. In one group they were kept one by one in little food vials ("solitary"); in the other they were allowed to live socially in the flight cage ("cage flies") and were repeatedly treated with various stimulants. After 


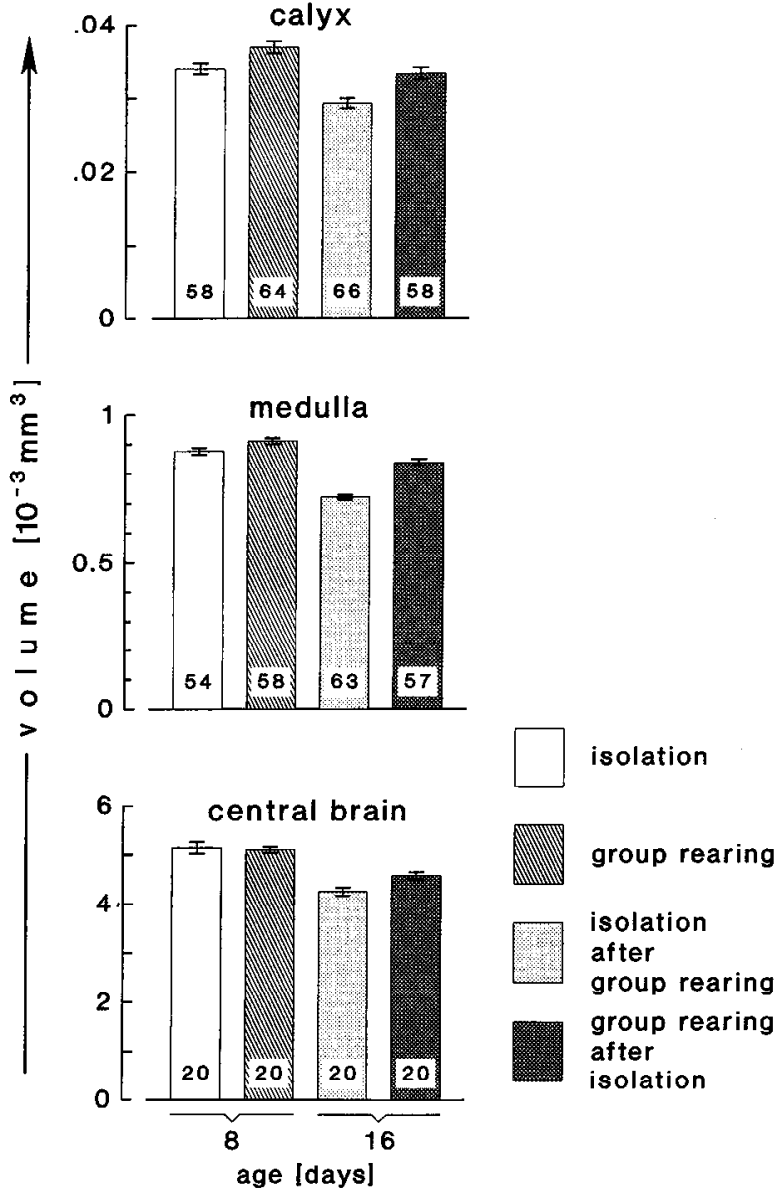

Figure 5. Structural changes are not confined to a "critical" period early in imaginal life. Flies were kept singly or under "enriched" conditions as described in the legend to Figure 4 and in the text. After 8 $d$, half of them were sacrificed for histology. The others were transferred to the alternative conditions for another $8 \mathrm{~d}$. Calyx, medulla, and central brain without optic lobes were evaluated from 8- and 16-d-old females. $n$ indicates the number of neuropil regions measured. After $8 \mathrm{~d}$, differences are significant for calyx and medulla $(p<0.05)$. The highly significant differences $(p<0.001)$ after $16 \mathrm{~d}$ in all three neuropil regions reflect the conditions of the second half period

that time, all flies were immobilized by cooling to $5^{\circ} \mathrm{C}$, and half of the females from either group were sacrificed for histology; the remaining animals were subjected to the opposite treatment for another $8 \mathrm{~d}$ : flies that previously had been kept solitary now were placed into the cage, and vice versa. Only the calyx, medulla, and central brain were evaluated. Although all flies sacrificed after $16 \mathrm{~d}$ had spent as much time in isolation as in the flight cage, their brain volume clearly reflected the treatment during the 2 nd $8 \mathrm{~d}$ period, and volume differences were about as large as in the previous experiment. Secondly, comparison of the brains of the flies sacrificed after 8 and $16 \mathrm{~d}$ showed a general volume loss between the 8th and 16th d, superimposed upon the differential effects caused by the two types of living conditions. Since this overall decrease had not been observed in studies without transfer (and, hence, without cold anesthesia), conceivably the general reduction in volume was a consequence of this treatment. No control experiment was performed to test this hypothesis. Thirdly, volume differences in the calyx and medulla scemed to develop at a different rate. After $8 \mathrm{~d}$, the difference in the medulla was small and hardly significant, whereas in the calyx it was already substantial (9\%). This could be taken as an indication that different cellular processes are underlying the volume changes (and, concomitantly, their functional role in behavior) in these two brain regions.

What causes the volume changes? Technau (1984) had considered olfactory stimuli or/and social contact the likely factors influencing fiber number in the mushroom bodies. We reinvestigated this question and compared four types of living conditions: (1) isolation in small food vials as described above; (2) normal mass culture in standard food bottles; (3) mass culture in an empty flight cage with nothing but standard food in an open food bottle; (4) mass culture in the flight cage with full treatment as described above. This experiment yielded two surprising results (data not shown): the brains of flies kept for 19 d (compare experiment of Fig. 4) under the normal rearing conditions in our standard food bottles were as small as those of flies kept in isolation in small plastic vials. Secondly, the flight cage with nothing but an open food bottle was as good in supporting large brains as was the cage with the full treatment. Apparently, tlies needed enough space. Social contact under crowded conditions was not enough to support the development or maintenance of large brains. In fact, our experiments did not indicate that it even contributed at all to increased brain size.

Since the results, if true, had far-reaching consequences, we decided to repeat some of the experiments with slight variations, despite the enormous labor involved in each one. They came out differently and further repetitions again gave new results. The following 18 months of futile experimentation made clear that brain size was influenced by more than one uncontrolled variable. What remained undisputable at this stage of the investigation was that (1) most neuropil regions can dramatically change in volume depending on living conditions; (2) these changes occur in different brain regions to different degrees; (3) they are not confined to the first week of adult life; and (4) they do not depend upon experimentally provided olfactory stimuli.

Volume changes in a controlled atmosphere. In the following, several basic features of the experimental conditions were modified in order to kecp all paramctcrs as constant as possible. It has been shown above that already 1 week of adult life is enough to influence brain size substantially. Therefore, the exposure to the respective living conditions was restricted to the first week of imaginal life to avoid influences of bacterial and fungal growth. Secondly, all flies were kept in a controlled atmosphere to eliminate some of the seasonal cues. Air from a central compressor was passed through a charcoal filter and two wash bottles. As before, cultures were kept at high constant humidity, constant temperature, and a controlled light:dark cycle.

In the first series of experiments in the spring and late summer of 1993, flies were either kept one by one in small food vials ("solitary") or as heterosexual groups of 40 flies in $70 \mathrm{ml}$ food bottles ("group flies"). Each experiment was exactly replicated right after the first one (i.e., 1 week later) to test repeatability. In a second set of experiments in October 1993, flies of heteroand homosexual pairs were compared. Finally, in December 1993 and March 1994, solitary flies were compared with cage flies (i.e., kept directly in the flight cage) in heterosexual groups of 500 flies. Results are documented in Table 1 and Figures 610.

First, the data obtained between March and December 1993 show an unexpected trend toward smaller volumes both in the calyx and the lobula (Fig. 6). So far, we have no explanation for this reduction. An artifact of data acquisition due to increasing 
Table 1. Volume of lobula and calyx in 7-d-old flies kept under different living conditions

\begin{tabular}{lllllllr} 
Date & $\begin{array}{l}\text { Living } \\
\text { conditions }\end{array}$ & Sex & $\begin{array}{l}\text { Lobula } \\
\text { (SEM) }\end{array}$ & $n$ & $\begin{array}{l}\text { Calyx } \\
\text { (SEM) }\end{array}$ & $n$ \\
\hline A & & & & & & \\
$3-19-93$ & solitary & f & $334.5(4.9)$ & 46 & $34.01(0.69)$ & 78 \\
& group & f & $329.3(5.7)$ & 40 & $33.40(0.75)$ & 66 \\
& solitary & m & $367.3(4.8)$ & 54 & $39.18(0.52)$ & 98 \\
& group & m & $349.4(4.6)$ & 32 & $38.04(0.62)$ & 58 \\
$3-26-93$ & solitary & f & $332.4(4.4)$ & 40 & $35.66(0.53)$ & 94 \\
& group & f & $322.9(3.1)$ & 44 & $33.43(0.58)$ & 82 \\
& solitary & m & $389.2(5.5)$ & 46 & $39.32(0.59)$ & 78 \\
& group & m & $357.5(4.0)$ & 44 & $38.72(0.55)$ & 62 \\
$7-27-93$ & solitary & f & $302.4(3.9)$ & 46 & $31.47(0.53)$ & 92 \\
& group & f & $273.4(3.5)$ & 48 & $28.51(0.45)$ & 102 \\
& solitary & m & $315.2(4.3)$ & 48 & $35.13(0.47)$ & 102 \\
& group & m & $291.5(3.7)$ & 46 & $33.40(0.42)$ & 94 \\
8-6-93 & solitary & f & $306.8(4.4)$ & 50 & $30.30(0.56)$ & 92 \\
& group & f & $288.4(3.6)$ & 46 & $28.24(0.67)$ & 92 \\
& solitary & m & $348.3(4.5)$ & 52 & $36.39(0.40)$ & 96 \\
& group & m & $326.9(4.2)$ & 52 & $36.15(0.52)$ & 96 \\
B $10-15-93$ & with fem. & f & $291.7(2.8)$ & 68 & $30.41(0.52)$ & 86 \\
$10-7-93$ & with fem. & f & $286.4(2.6)$ & 76 & $29.79(0.57)$ & 90 \\
& with male & f & $293.1(3.8)$ & 64 & $29.13(0.45)$ & 98 \\
& with male & m & $321.5(3.9)$ & 88 & $33.70(0.49)$ & 104 \\
& with male & f & $281.4(3.9)$ & 54 & $28.67(0.51)$ & 94 \\
& with male & m & $328.4(3.1)$ & 86 & $34.14(0.42)$ & 106 \\
& with fem. & m & $324.8(3.7)$ & 84 & $34.57(0.45)$ & 100
\end{tabular}

C

$\begin{array}{llllr}12-17-93 & \text { solitary } & \text { f } & 279.4(3.4) & 70 \\ & \text { cage } & \text { f } & 299.9(3.2) & 70 \\ & \text { solitary } & \text { m } & 295.1(3.4) & 74 \\ & \text { cage } & \text { m } & 294.6(3.4) & 74 \\ 3-11-94 & \text { solitary } & \text { f* }^{*} & 296.6(2.8) & 146 \\ & \text { cage } & \text { f* }^{*} & 311.2(2.6) & 138 \\ & \text { solitary } & \text { m}^{*} & 316.5(3.0) & 148 \\ & \text { cage } & \text { m}^{*} & 317.5(3.2) & 146\end{array}$

Flies are kept singly or in defined groups under standardized conditions during the first week of adulthood. Subsequently, they are sacrificed for histology. The volumes of their lobulae and calyces are measured planimetrically. Date indicates the time of eclosion from the pupal case. Living conditions are specified in the text. Volumes are given in $10^{-6} \mathrm{~mm}^{3} ; n$ is the number of neuropil bodies recorded. Note that the results presented in Figures 6-10 are based on the data of Table 1, as indicated in the legends to the figures. The experiment of 1994 (marked with an asterisk in the column "Sex") was performed with a new line of wild-type Berlin generated three generations earlier by mixing equal numbers of flies from the respective Würzburg and Tübingen stocks.

experience in planimetry has been excluded by remeasuring the preparations. We consider four types of causes: (1) a circannual cycle; (2) genetic "degeneration" of the stock; (3) a gradually changing level of pollutant from the new building (e.g., poison in the water, dust in the air); (4) a gradual change in some parameter of the histological procedure (e.g., deteriorating hot plate, paraffin bath, etc.). Recently, in March 1994, a further experiment was performed with a new line of wild-type Berlin (WTB) obtained by pooling the Würzburg and Tübingen lines of WTB that had been separated for the last 15 years. With flies of this stock, lobula volume had returned to previous values

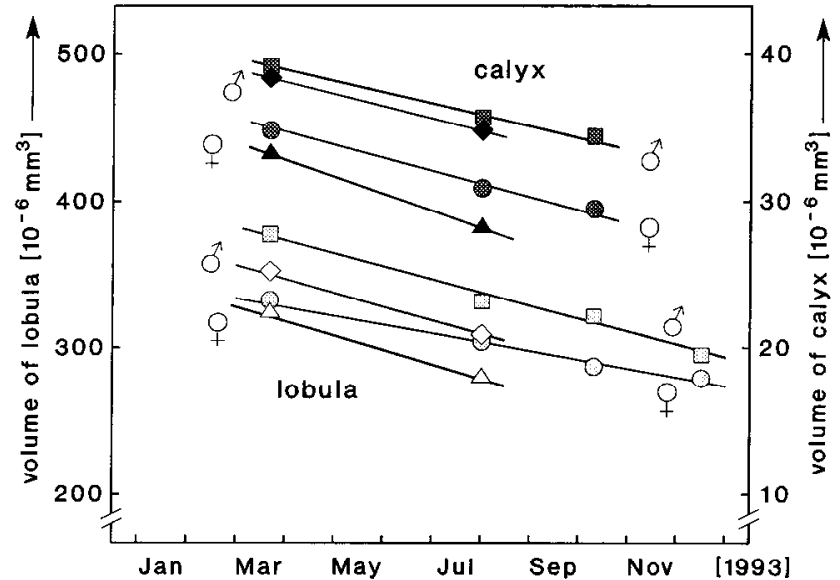

Figure 6. A steady decline in the volume of the calyx (upper set of data; scale on the right) and of the lobula (lower data set; scale on the left) of wild-type Berlin during 1993. Volume measurements on solitary flies (light and dark gray circles and squares) as well as on flies kept in heterosexual groups of 40 individuals (black and white diamonds and triangles) are shown (see also Fig. 8). The October data are from flies kept as homo- or heterosexual pairs (Fig. 9). In December, only lobulae were evaluated (Fig. 10). Note that males (squares and diamonds) have larger calyces and lobulae than the corresponding females (circles and triangles). Data from identical experiments performed 1 week apart were pooled except for the experiment of December, which was not replicated immediately. For "raw" data and sample sizes $(n)$, see Table 1.

(data not shown in Fig. 6). If substantiated, this result supports hypothesis (1) and (2). If the same effect should be found with the "old" WTB stock, we would be faced with a circannual cycle; if not, genetic "degeneration" would be the most likely (but not the only) explanation. This experiment is in progress.

Reproducibility from one week to the next is still problematic. In 7 of the 24 pairs of measurements taken under seemingly identical conditions, the two values differ significantly (Fig. 7). All of them are positive, meaning that the value of the second week is larger than that of the first, against the long-term trend. Even the nonsignificant differences show this bias. Five of the significant differences belong to a block of eight data points taken in one pair of experiments in late July/early August, suggesting a specially severe problem at this date. We conclude that not all parameters influencing brain size are yet under control, and that one of them systematically changes between the first and second week of experimentation.

In accord with previous data, the 1993 experiments show that in males the lobula is about $10 \%$, and the calyx 15-20\% larger than in females (Table 1, Fig. 6). Even the medulla is larger in males than in females, although this difference is only $3-4 \%$ (data not shown). The larger optic lobes of males overcompensate the larger number (about $5-10 \%$ ) of optic lobe columns females have due to the larger number of ommatidia in their eyes. Male optic lobes may house a special fiber system for the visual component of male courtship tracking, as exists in calliphorid and muscid flies (Strausfeld, 1980, 1991; Hausen and Strausfeld, 1980). The larger male calyx is surprising, given the observation of Technau (1984) that the peduncle contains more Kenyon cell fibers in the female than in the male.

The difficulties with reproducibility require that in experiments in which the influence of living conditions ("experience") are studied, groups of sibling flies are compared side by side. In the first experiment mentioned above, solitary and group flies 


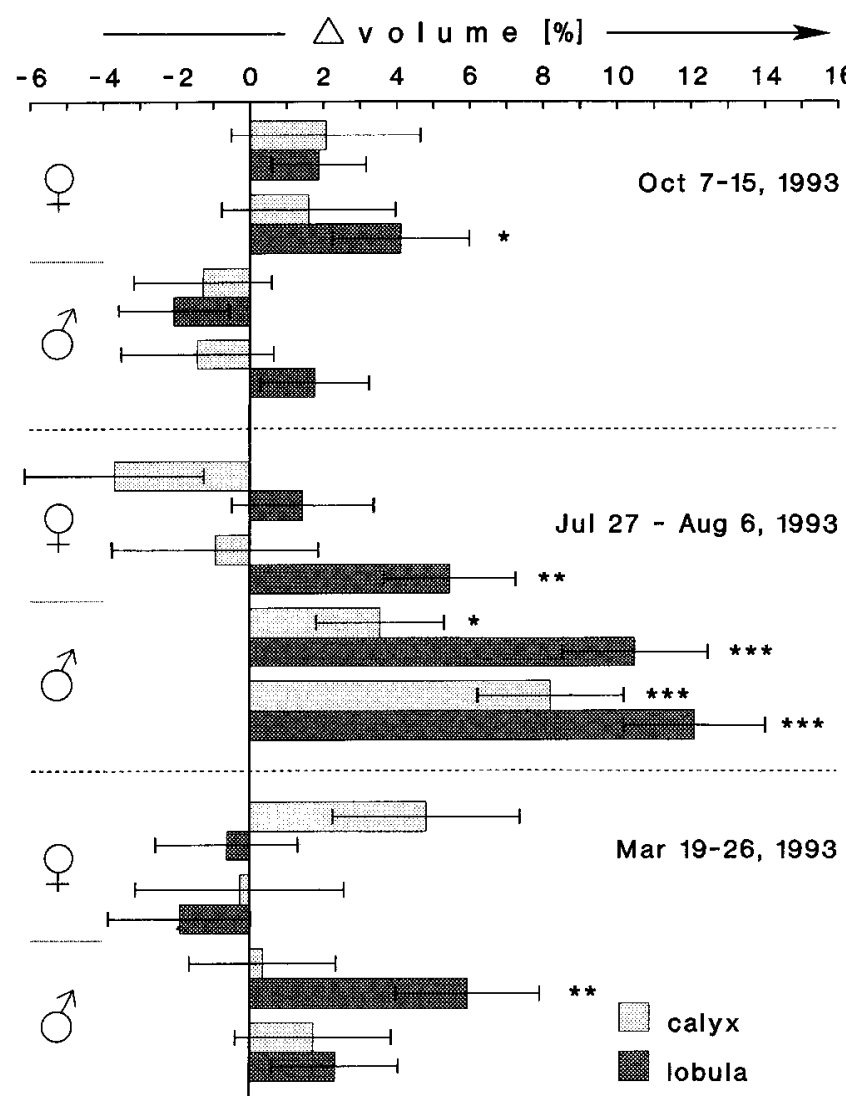

Figure 7. Reproducibility of results from 1 week to the next. Each bar shows the difference between two measurements taken 1 week apart under the same experimental conditions. Positive (negative) volume differences indicate that in the second experiment a larger (smaller) volume was found than in the first. Note a positive tendency throughout which is particularly large in the July/August data. SEMs of the differences are derived by error propagation from the SEMs of the two respective volume measurements of which the difference was calculated. Sample sizes $(n)$ are given in Table 1, parts A and B. Significance levels are indicated by asterisks. ${ }^{*} p<0.05 ;{ }^{* *} p<0.01 ;{ }^{* *} p<0.001$.

of mixed sex were kept in small food vials in the same incuhator. Flies were transferred to new vials after $3 \mathrm{~d}$ and sacrificed for histology after $7 \mathrm{~d}$. Group flies of both sexes developed about $5 \%$ smaller lobulae and calyces than solitary flies (Fig. 8). The experiment was performed four times, twice in March, once in July and August. The outcome was remarkably similar (Table 1, part A).

What experimental factor is it that actually causes these volume differences? Is it stress, locomotor activity, social contact, or the available space? Or could it be the chemical environment of the flies? The solitary flies live each one in $12.5 \mathrm{ml}$ of air that is exchanged but slowly against the air of the incubator. They have $3.1 \mathrm{~cm}^{2}$ of food surface at their disposition. The 40 group fiies together have about $40 \mathrm{ml}$ of air space and $8.5 \mathrm{~cm}^{2}$ of food surface available. To minimize influcnces of the food and air, we compared the brains of flies that were kept in pairs of the same or opposite sex in small vials (Fig. 9; Table 1, part B). Females in the company of a female have about $4 \%$ larger calyces than females in the company of a male. Likewise, a small though just not significant effect in the same direction is observed for males. The lobula seems not to be differentially affected by the sex of the companion. Nevertheless, the results indicate that social contact has an effect on brain size.

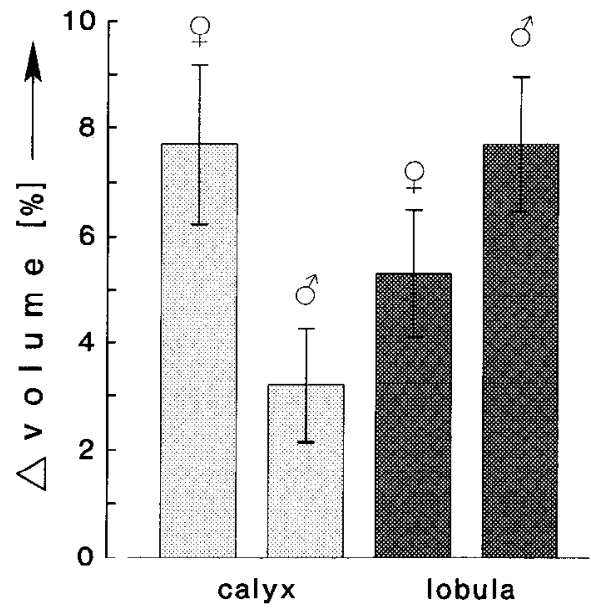

Figure 8. Volume differences of calyx and lobula betwecn flics kept for 1 week singly in small food vials and flies that had passed the same time in heterosexual groups of 40 animals in medium-sized food bottles. Note that (in contrast to Figs. $4,5,10$ ) positive values indicate that solitary flies had larger volumes of neuropil than group flies. Data are averages of four independent measurements from March and July/August 1993 (Table 1, part A; Figs. 6, 7). Sample sizes $(n)$ are given in Table 1, part A. For SEMs, see legend to Figure 7. All differences are highly significant $(p<0.001$, except male calyx, for which $p<0.01)$.

In a further experiment, solitary flies were compared with flies kept directly in the flight cage (cage flies) in heterosexual populations of about 500 animals. Open food vials $(180 \mathrm{ml})$ were provided in the cage and were exchanged every day. This should keep the chemical environment for both groups as similar as possible. At the end of the first week of imaginal life, females directly from the cage have about $7 \%$ larger lobulae than solitary females (Fig. 10). 'This is what we had observed already earlier. It is a remarkable result since group flies in medium-sized food vials have smaller lobulac than solitary flics (sce Fig. 8). Wc conclude that not only social contact but also the available living space has an influence on brain structure. In males, the lobula

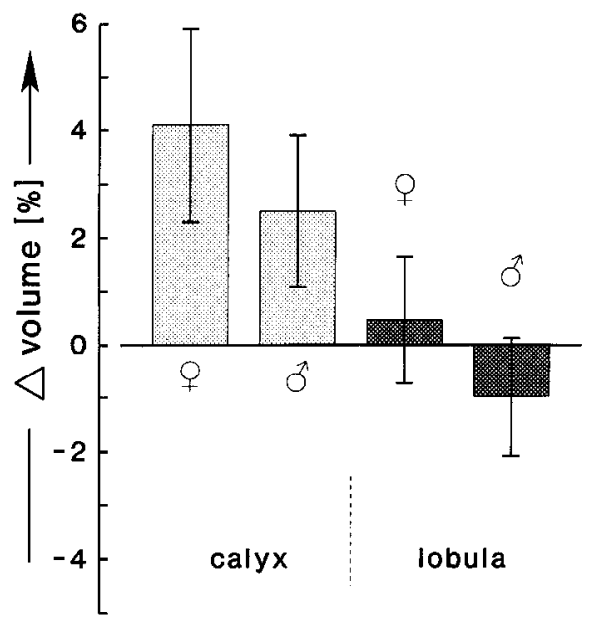

Figure 9. Sex of partner influences size of calyx, but not lobula. Flies are kept for 1 week in homo- or heterosexual pairs in small food vials. Positive difference indicates that presence of a female leads to larger volume of neuropil than the presence of a male. Difference is significant $(p<0.05)$ for female calyx and just not significant $(p<0.1)$ for male calyx. No significant differences are found for lobula. Volume data and sample sizes $(n)$; see Table 1, part B, for SEMs, see legend to Figure 7. 


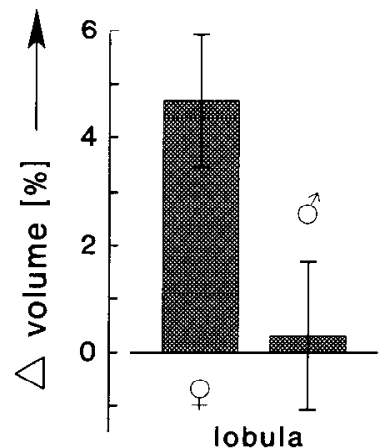

Figure 10. Volume differences of lobula between solitary flies confined for I week to small food vials and flies kept in a flight cage in a hetcrosexual group of about 500 animals. In contrast to the experiments of Figures 4 and 5, the flight cage contained no plants or odorants, only an open food bottle. (The closed vials of the solitary flies were also placed into the cage to provide them with a similar atmosphere.) No handling was applied to either group. Female cage flies have significantly larger lobulae $(p<0.001)$ than their solitary female siblings. For males, no difference is observed. This experiment was conducted once in December 1993, once in March 1994. Data are pooled although overall volumes in the second experiment (new wild-type Berlin) were larger than in the first. For sample sizes see Table 1, part C, for SEMs, legend to Figure 7.

of solitary and cage flies in this experiment has about the same volume. Apparently, the large space of the cage just compensates for the group effect. Interestingly, in the flight cage, the males are found crowded near the food site most of the time, whereas the females are dispersed in the cage and come to the food only for discrete periods.

\section{Discussion}

There can be little doubt that invertebrates, including insects, possess the means for experience-dependent structural plasticity of the nervous system (Technau, 1984; Atwood and Govind, 1990; Budnik et al., 1990; Bailey and Kandel, 1993; Withers et al., 1993). What is less clear is, to what extent such mechanisms affect the structure of the brain. We have demonstrated that brain size in Drosophila is extremely variable, that much of this plasticity is experience dependent, and that different experiences affect the various brain regions differentially. Let us now return to, and reexamine the three questions raised in the introductory remarks: What happens at the cellular level? What are the decisive factors in the environment? What are the behavioral correlates of the structural changes?

\section{Cellular mechanisms}

No doubt, the observed volume changes must be brought about by changes in the size or number of cells. Before discussing the cellular level, one should reconsider possible artifacts. In this study, brain size is determined in histological preparations that go through a long series of treatments before they can be evaluated. Most of these steps influence the final size of the tissue, and some of them cannot easily be quantified. It seemed crucial, therefore, to use the mass histological procedure and to compare only groups of which the brains had been processed side by side in the same collars and on the same microscope slides. The discussion of experience-dependent effects will be confined entirely to differences obtained in this way.

On the other hand, since in most experiments four groups are compared and only five heads of each group fit on one micro- scope slide, 15 to 20 microscope slides are evaluated in each experiment. This number should be large enough to provide an average for the variability in handling, in particular, since the mass histological procedure is a daily routine in the laboratory. In fact, the data do not indicate much variability in handling. There is little scatter in the data revealing the negative trend over the year 1993 (Fig. 6). Moreover, handling should affect the eight measurements (calyx and lobula in four groups of animals) in one experiment alike. The variability from one week to the next (Fig. 7) does not indicate such an effect. For instance, in the experiment of July/August 1993, the large difference in brain size between the first and second week seems to affect only the males and not the females, although both sexes were processed together. A male specific effect is likely to be produccd in vivo, probably by a bchaviorally relevant factor. In the two experiments performed 1 week apart, there is a consistent tendency for the measurements of the second week to come out slightly larger than those of the first one. A handling artifact cannot be excluded. However, it should be kept in mind that between the first and the second experiment the incubator was not cleaned and the charcoal filter not renewed. Even in this case, in vivo effects are a likely explanation.

Again, the downward trend during 1993 may be an artifact. It might, for instance, reflect an aging thermostat or a deteriorating chemical. Our new data of 1994 argue against this interpretation. Given the extreme sensitivity of the living brain to conditions that influence its size, one should consider a circannual trend in the flies' environment such as the $\mathrm{CO}_{2}$ concentration in the air (e.g., Stange, 1992), which would not be eliminated in the "controlled" atmosphere of our incubator.

We now turn to the size differences between brains of sibling flies processed together, and seek the underlying cellular mechanisms. Presumably, several independent processes of structural reorganization are at work serving different functions. It may, indeed, be a specialty of the mushroom bodies that the Kenyon cells can shed their fibers and grow new ones. This property is particularly obvious at the onset of metamorphosis, since at that stage massive fiber decay and regrowth can be observed directly. During adulthood this process may well continue to operate, though in a desynchronized manner. It is not clear how experience would influence it. It has been proposed that this fiber outgrowth and decay might be involved in the formation and refinement of odor templates via a selection process (Heisenberg, 1989, 1994).

Cell bodies of incompletely differentiated neurons that send out fibers can not be the explanation of volume changes in the optic lobes. Most of the neurons contributing to the lamina cartridges and to the lamina-to-medulla projection are thought to be known (Trujillo-Cenóz, 1965; Boschek, 1971; Fischbach and Dittrich, 1989) and to occur precisely once per cartridge and medulla column. We, therefore, consider it unlikely that in the optic lobe entire arbors of neurons are added or removed. Changes in the size and number of terminal branches, swelling, or spinc formation and retraction arc morc likely to be responsible for these differences. Addition of tracheae or swelling of glia must also be taken into account.

For the housefly Musca domestica, Meinertzhagen and coworkers (Kral and Meinertzhagen, 1989; Rybak and Meinertzhagen, 1990) have demonstrated that size and frequency of synapses in the lamina depend upon the illumination regime. Short exposures to light after extended periods in the dark at all ages lead to an increased proportion of small synapses. It would 
bc most intercsting to extend these findings to Drosophila and to relate them to the volume changes reported in the present study. The point to be made here is that a variety of different processes, inductive and selective, may be at work in different parts of the brain modifying its fine structure. Volume measurements, unfortunately, cannot document the local structural dynamics at the network level.

Of the various possible cellular effects underlying the volume changes, the least interesting would be a uniform swelling or shrinkage of all or most neurons in the brain. This hypothesis is not supported by the data. In the homosexual and heterosexual pairs, for instance, the calyx but not the lobula is differentially affected. The same partial independence of calyx and lobula is apparent in the comparison of solitary and group flies. If the effect were a general swelling or shrinkage of processes (due to a single variable such as salt concentration in the hemolymph), the different regions of the brain should be affected at a fixed ratio in the various experiments.

\section{Which factors in the fly's environment cause the volume changes?}

It is tempting to invoke psychological entities such as stress, individual living space, social contact, etc., as causative factors in volume changes. These terms may be heuristically useful in the design and interpretation of the experiments. But eventually they will have to be analyzed in more objective terms if they are to be measured and if their meaning with respect to flies is to be made more evident. Individual contributions of specific sensory stimuli, specific memories, physical space or locomotor activity need to be isolated.

A remarkable result of this study is that well-defined biologically relevant differences in the environment of a fly have measurable effects on a certain region of neuropil. Females after 1 week together with another female have larger calyces than after a week in the company of a male. An individual's life experience can, thus, be encoded in the volume of selected neuropil regions. Sexual activity is known to affect metabolism and longevity (Partridge et al., 1987; Giesel et al., 1989). After copulation, females intensify egg production. It is conceivable that these metabolic processes might compete for resources that are also required for Kenyon cell fiber formation, so that the latler process would be slowed down. We prefer to believe, though, that independent of metabolic rates, sexual activity has profound consequences for the structure of the brain, with a net effect on volume in the female calyx but not in the other regions measured.

The observation that space may be a crucial parameter needs further investigation. The available amount of space may affect sensory processing and locomotor activity. From current evidence, we cannot distinguish between these two. We have tested three conditions that differ each by about one order of magnitude from the ncxt one in the average space per fly $\left(1 \mathrm{~cm}^{3}\right.$ for group flies, $12.5 \mathrm{~cm}^{3}$ for solitary flies, $250 \mathrm{~cm}^{3}$ for cage flies). The volume of the lobula follows this trend. Surely, the different amount of space available must have a profound effect on a fly, but whether "space per fly" is behaviorally relevant remains an open question. The relation between living space, locomotor activity, and the volume of the optic lobes needs to be further explored. It will now be important to monitor directly the longterm locomotor behavior under the conditions used.
Bchavioral correlates: are these structural changes memory traces?

The answer, of course, depends on how narrowly we define "memory traces." Evidently, the structural modifications must change the functional properties of the network they concern even if only in subtle ways. Changes in the lamina can only directly affect processing steps taking place at this level although this, in turn, will have consequences at other levels. Therefore, it may be very instructive to try to modify the lamina or the lobula plate or any other neuropil using specific, well-defined stimuli. Probably the neuronal circuitry is constantly being optimized for those tasks that are currently important. Under some of our differential conditions changes occur about everywhere in the brain. This does not imply that memory traces of specific learning tasks are distributed everywhere. Flies may learn a variety of things by a variety of cellular and molecular mechanisms during the $7 \mathrm{~d}$ of exposure.

Whatever the particular behavioral correlates of the observed volumc changes may bc, our investigation suggests that not only in vertebrates but also in arthropods sprouting and decay of terminal branches as well as the concomitant turnover and reorganization of synapses occur continuously and in many, if not all, regions of the brain.

\section{References}

Ashburner M (1989a) Drosophila-a laboratory handbook, pp 192194. Cold Spring Harbor, NY: Cold Spring Harbor Laboratory.

Ashburner M (1989b) Drosophila-a laboratory manual, pp 254-259. Cold Spring Harbor, NY: Cold Spring Harbor Laboratory.

Atwood HL, Govind CK (1990) Activity-dependent and age-dependent recruitment and regulation of synapses in identified crustacean neurons. J Exp Biol 153:105-127.

Bailey CH, Kandel ER (1993) Structural changes accompanying memory storage. Annu Rev Physiol 55:397-426.

Balling A, Technau G, Heisenberg M (1987) Are the structural changes in adult Drosophila mushroom bodies memory traces? Studies on biochemical learning mutants. J Neurogenet 4:65-73.

Boschek CB (1971) On the fine structure of the peripheral retina and the lamina of the fly Musca domestica. Z Zellforsch 110:336-349.

Budnik V, Wu CF, White K (1989) Altered branching of serotonincontaining neurons in Drosophila mutants unable to synthesize serotonin and dopamine. J Neurosci 9:2866-2877.

Budnik V, Zhong Y, Wu CF (1990) Morphological plasticity of motor axons in Drosophila mutants with altered excitability. J Neurosci 10: 3754-3768.

Corfas G, Dudai Y (1991) Morphology of a sensory neuron in Drosophila is abnormal in memory mutants and changes during aging. Proc Natl Acad Sci USA 88:7252-7256.

Coss RG, Brandon JG (1982) Rapid changes in dendritic spine morphology during the honeybee's first orientation flight. In: The biology of social insects (Beed MD, Michener CD, Evans HE, eds), pp 338342. Boulder, CO: Westview.

Cotman CW. Gibbs RG, Nieto-Sampedro M (1987) Synapse turnover in the central nervous system. In: The neural and molecular bases of learning (Changeux JP, Konishi M, eds), pp 375-398. New York: Wiley.

deBelle S, Heisenberg M (1994) Associative odor learning in Drosophila abolished by chemical ablation of mushroom bodies. Science 263:692-695.

Durst C, Eichmüller S, Menzel R (1993) Experience dependent volume changes in honeybee mushroom body. In: Gene-brain-behavior. Proceedings of the 21 st Göttingen neurobiology conference (Elsner N, Heisenberg M, eds), Abstr 777. Stuttgart: Thieme.

Erber J, Masuhr T, Menzel R (1980) Localization of short-term memory in the brain of the bee, Apis mellifica. Physiol Entomol 5:343358.

Fischbach KF, Dittrich APM (1989) The optic lobe of Drosophila melanogaster I. A Golgi analysis of wild-type structure. Cell Tissue Res 258:441-475. 
Giesel JT, Lanciani CA, Anderson JF (1989) Metabolic rate and sexual activity in Drosophila simulans. J Insect Physiol 35:893-895.

IIausen K, Strausfeld NJ (1980) Sexually dimorphic interneuron arrangements in the fly visual system. Proc R Soc Lond [Biol] 208:5771.

Heisenberg M (1989) Genetic approach to learning and memory (mnemogenetics) in Drosophila melanogaster. Fortsch Zool 37:3-45.

Heisenberg M (1994) Central brain function in insects: genetic studies on the mushroom bodies and central complex in Drosophila. Fortsch Zool 39:30-39.

Heisenberg M, Böhl K (1979) Isolation of anatomical brain mutants of Drosophila by histological means. Z Naturforsch [C] 34c:143147.

Heisenberg M, Borst A, Wagner S, Byers D (1985) Drosophila mushroom body mutants are deficient in olfactory learning. J Neurogenet 2:1-30.

Ito K, Hotta Y (1992) Proliferation pattern of postembryonic neuroblasts in the brain of Drosophila melanogaster. Dev Biol 149:134148.

Kral K, Meinertzhagen IA (1989) Anatomical plasticity of synapses in the lamina of the optic lobe of the fly. Philos Trans R Soc Lond [Biol] 323:155-183.

Menzel R, Hammer M, Braun G, Mauelshagen J, Sugawa M (1991) In: Neurobiology of learning and memory in honeybees (Fisher LJ, Goodmann RC, eds), pp 323-353. Wallingford, UK: CAB International.

Partridge L, Green A, Fowler K (1987) Effects of egg-production and of exposure to males on female survival in Drosophila melanogaster. J Insect Physiol 33:745-749.

Prokop A, Technau GM (1994) Normal function of the mushroom body defect gene of Drosophila is required for the regulation of the number and proliferation of neuroblasts. Dev Biol 161:321-337.

Rybak J, Meinertzhagen IA (1990) Experience-dependent changes in the sizes and frequencies of photoreceptor synapses in the fly's optic lamina. In: Brain-perception-cognition, Proceedings of the 18th Göttingen ncurobiology conference (Elsner N, Roth G, eds), Abstr 196. Stuttgart: Thieme.

Sang JH (1949) The ecological determinants of population growth in Drosophila culture. IV. Larval and pupal survival. Physiol Zool 22: 183-202.

Stange G (1992) High resolution measurement of atmospheric carbon dioxide concentration changes by the labial palp organ of the moth Heliothis armigera (Lepidoptera: Noctuidae). J Comp Physiol [A] 171:317-324.

Strausfeld NJ (1976) Atlas of an insect brain. Berlin: Springer.

Strausfeld NJ (1980) Male and female visual neurones in dipterous insects. Nature 283:381-383.

Strausfeld NJ (1991) Structural organization of male-specific visual neurons in calliphorid optic lobes. J Comp Physiol 169:379-393.

Technau G (1984) Fiber number in the mushroom bodics of adult Drosophila melanogaster depends on age, sex and experience. J Neurogenet $1: 113-126$.

Technau G, Heisenberg M (1982) Neural reorganization during metamorphosis of the corpora pedunculata in Drosophila melanogaster. Nature 295:405-407.

Thompson RF (1987) Activity-dependence of network properties. In: The neural and molecular bases of learning (Changeux JP, Konishi M, eds), pp 473-502. New York: Wiley.

Trujillo-Cenóz O (1965) Some aspects of the structural organization of the intermediate retina of dipterans. J Ultrastruct Res 13:1-33.

Withers GS, Fahrbach SE, Robinson GE (1993) Selective neuroanatomical plasticity and division of labour in the honey-bee. Nature 364:238-240.

Zhong Y, Budnik V, Wu CF (1992) Plasticity in Drosophila memory and hyperexcitable mutants: role of cAMP cascade. J Neurosci 12 . $644-651$. 